Turbulent contributions to Ohm's law in axisymmetric magnetized plasmas

I. Chavdarovski and R. Gatto

Citation: Physics of Plasmas 24, 072512 (2017); doi: 10.1063/1.4993208

View online: http://dx.doi.org/10.1063/1.4993208

View Table of Contents: http://aip.scitation.org/toc/php/24/7

Published by the American Institute of Physics

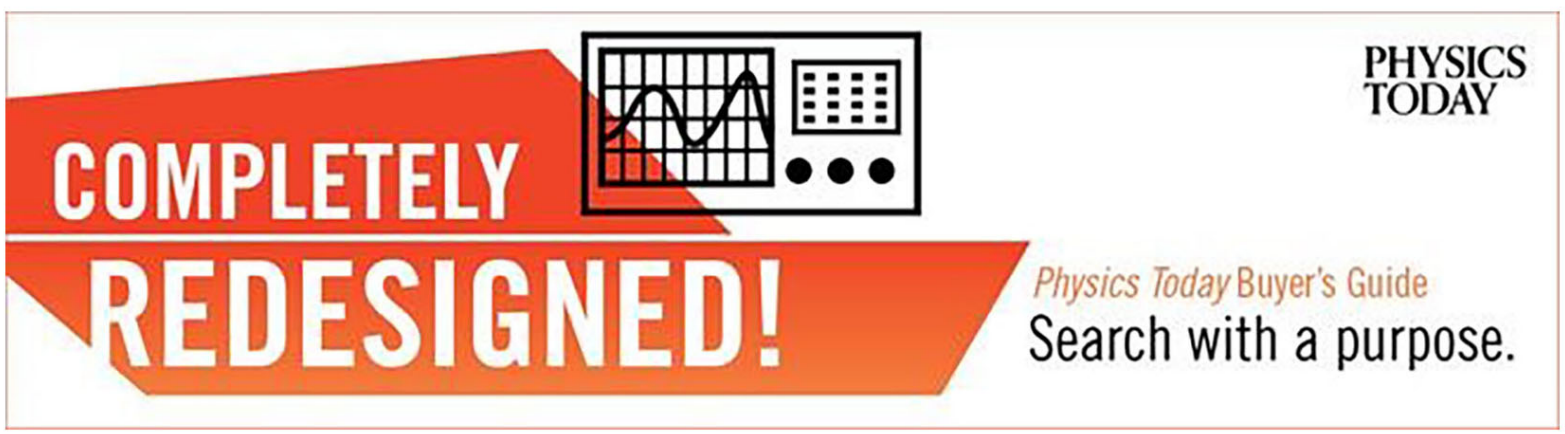




\title{
Turbulent contributions to Ohm's law in axisymmetric magnetized plasmas
}

\author{
I. Chavdarovski ${ }^{1}$ and R. Gatto ${ }^{2}$ \\ ${ }^{1}$ Max Planck Institute for Plasma Physics, Boltzmannstrasse 2, Garching 85748, Germany \\ ${ }^{2}$ Department of Astronautical, Electrical and Energy Engineering, Sapienza University of Rome, Italy
}

(Received 25 April 2017; accepted 27 June 2017; published online 13 July 2017)

\begin{abstract}
The effect of magnetic turbulence in shaping the current density in axisymmetric magnetized plasmas is analyzed using a turbulent extension of Ohm's law derived from the self-consistent action-angle transport theory. Besides the well-known hyper-resistive (helicity-conserving) contribution, the generalized Ohm's law contains an anomalous resistivity term and a turbulent bootstrap-like term proportional to the current density derivative. The numerical solution of the equation for equilibrium and turbulence profiles characteristic of conventional and advanced scenarios shows that, through the "turbulent bootstrap" effect and anomalous resistivity, power and parallel current can be generated which are a sizable portion (about 20\%-25\%) of the corresponding effects associated with the neoclassical bootstrap effect. The degree of alignment of the turbulence peak and the pressure gradient plays an important role in defining the steady-state regime. In a fully bootstrapped tokamak, the hyper-resistivity is essential in overcoming the intrinsic limitation of the hollow current profile. Published by AIP Publishing.
\end{abstract}

[http://dx.doi.org/10.1063/1.4993208]

\section{INTRODUCTION}

International research efforts on achieving the necessary conditions for controlled thermonuclear fusion in magnetically confined plasmas have shown that in order for a steadystate tokamak reactor to be economically attractive, a high fraction of the current needs to be generated non-inductively. The low drive efficiencies of the known external noninductive methods, such as radio-frequency-wave or neutral beam injection, increase the interest in regimes in which the current is generated internally via the bootstrap mechanism. The bootstrap current is a parallel current driven by the radial pressure gradient through the pressure anisotropy in toroidal geometry. ${ }^{1}$ In an ideal case, a high pressure tokamak would be able to generate all of its current via the bootstrap effect. The obvious setback to this scenario is the fact that the bootstrap current vanishes around the axis, where the pressure profile is flat and trapped particle population is small. ${ }^{2}$ Since a tokamak with large bootstrap current is ordinarily unstable to tearing modes, it has been suggested that poloidal flux generated spontaneously near the edge by the dynamo effect induced by the turbulent perturbations can overcome this intrinsic limitation by diffusing the bootstrap current toward the center. This idea has been the driving force behind many theoretical works, ${ }^{3,4}$ as well as experimental campaigns aimed at achieving, via optimization of the plasma profiles, a steady-state "fully bootstrapped tokamak" operation. ${ }^{5-7}$

The role of turbulence in explaining the anomalous current diffusion observed in experiments is not yet clear. It has been shown in various theoretical works that turbulence leads to three additional terms in Ohm's law: (i) hyper-resistivity, a viscous-like term which induces current diffusion towards the axis, ${ }^{8}$ (ii) anomalous resistivity which adds to the neoclassical resistivity, ${ }^{9}$ and (iii) a bootstrap-like term ${ }^{10}$ which generates current density due to the transfer of linear momentum to the electrons at the expense of the energy in the turbulent perturbations. $^{11,12}$ The scope of this work is to identify scenarios in which the turbulent contributions play a significant role in regulating the current density profile and sustaining the equilibrium.

In this work, we present numerical studies based on a turbulent extension of Ohm's law ${ }^{10}$ derived in the framework of the action-angle ${ }^{13}$ self-consistent ${ }^{14}$ transport theory. The self-consistency implies that the collision operator contains both diffusion and drag in action-space, as opposed to the quasi-linear approach which includes only the diffusion part. The radial structure of the turbulent transport coefficients is presented, as a function of the magnetic turbulence and the thermodynamic equilibrium profiles. The hyper-resistive term is only related to the momentum transport, while the anomalous and cross-resistivity (bootstrap-like) coefficients contain also terms originating from the electron momentum source. The cross-resistive term leads to an amplification of the total current, while the anomalous resistivity and hyperresistivity lead to a current reduction. However, we show that the anomalous resistivity can increase significantly the current in the outer region and in some scenarios, like the advanced, even generate power from turbulence instead of dissipating it.

The present work continues the research line of Ref. 10, extending it to include advanced plasma regimes and fully bootstrapped tokamaks. In Sec. II, we give a very brief overview of the theoretical transport model and then present the turbulent electric field, referring the interested reader to Ref. 10 for the detailed derivation of it. The significance of various turbulent contributions is discussed in Sec. III where we present their radial dependence for different regimes and turbulence profiles. In Sec. IV, we give the final form of the turbulent Ohm's law and describe a power balance equation derived from it. A series of numerical studies that consider various thermodynamic profiles characteristic of L-mode 
regimes, advanced scenarios, and fully bootstrapped tokamaks are given in Sec. V. These studies clarify the potential role that the effect of turbulence has in shaping current density and safety factor profiles and show how turbulence can provide explanation to some experimental observations. The summary and the conclusions are presented in Sec. VI.

\section{THEORETICAL MODEL}

The parallel momentum transport equation stemming from the self-consistent action-angle transport theory is ${ }^{10}$

$$
\begin{aligned}
\mathcal{V}_{a} & \frac{\partial\left\langle M N(\mathbf{x} ; t) V_{\|}(\mathbf{x} ; t)\right\rangle_{\bar{r}}}{\partial t}-\mathcal{V}_{a}\left\langle q_{1} N E_{t}\right\rangle_{\bar{r}} \\
& +\frac{\partial}{\partial \bar{r}}\left\langle\mathcal{V}_{a} V_{f} M N V_{\|}\right\rangle_{\bar{r}} S(1-\kappa) \\
& +\frac{\partial}{\partial \bar{r}} \mathcal{V}_{a} \Gamma^{V}(\bar{r})=\left(\bar{r} B_{0, t}\right) \mathcal{V}_{a} U^{V}
\end{aligned}
$$

where $\mathcal{V}_{a}=4 \pi^{2} R_{0} w \bar{r}$ is the toroidal shell of width $w$ (centered at $\bar{r}$ ) over which magnetic perturbation mode $a$ generated by the bulk ions is nonzero, $r$ is a cylindrical radial coordinate defined through the toroidal flux $\Psi_{t}=\pi r^{2} B_{0, t}$, $E_{t}=(1 / c R)\left(\partial \psi_{p} / \partial t\right)$ is the induced toroidal electric field at a fixed position in space, while $\Gamma^{V}$ and $U^{V}$ account for the momentum transport and generation due to fluctuations, respectively. The third term on the LHS of Eq. (1) containing the velocity of a flux surface, $V_{f}=c E_{t} / B_{p}$, is a collisionless version of the Ware-Galeev pinch, effective only for trapped particles as described by the step function $S(1-\kappa)$, yielding 1 for trapped particles $(\kappa \in[0,1])$ and 0 for circulating particles $(\kappa \in(1,+\infty))$. Eq. (1) is a generalization of the quasilinear result of Ref. 15 .

The momentum flux and the momentum source present in Eq. (1) have been derived in Refs. 16, 17, and 18, respectively, with

$$
\Gamma_{e i}^{V}=-\mathcal{L}_{e i} \hat{\Gamma}_{2}^{e i} M_{e} \frac{d V_{\| e}}{d r}-\mathcal{L}_{e i} \hat{\Gamma}_{1}^{e i} \frac{M_{e} V_{\| e}}{\rho_{\mathrm{e}, \mathrm{p}}}
$$

and

$$
\left(r B_{0 t}\right) U_{e i}^{V}=-\mathcal{L}_{e i} \hat{U}_{2}^{e i} \frac{M_{e}}{\rho_{\mathrm{e}, \mathrm{p}}} \frac{d V_{\| e}}{d r}-\mathcal{L}_{e i} \hat{U}_{1}^{e i} \frac{M_{e} V_{\| e}}{\rho_{\mathrm{e}, \mathrm{p}}^{2}},
$$

where the following non-dimensional radial functions were introduced

$$
\begin{aligned}
& \hat{\Gamma}_{1}^{e i}=3 \rho_{\mathrm{e}, \mathrm{p}} {\left[\frac{1}{p_{e}} \frac{d p_{e}}{d r}+\frac{1}{p_{i}} \frac{d p_{i}}{d r}-2\left(\frac{T_{i}}{T_{e}}-\frac{3}{2}\right)\left(\frac{1}{q_{\mathrm{saf}}} \frac{d q_{\mathrm{saf}}}{d r}\right)\right] } \\
& \hat{\Gamma}_{2}^{e i}=3 \\
& \hat{U}_{1}^{e i}= 15 \rho_{\mathrm{e}, \mathrm{p}}^{2}\left(\frac{1}{q_{\mathrm{saf}}} \frac{d q_{\mathrm{saf}}}{d r}\right)^{2} \frac{T_{i}}{T_{e}}+3 \rho_{\mathrm{e}, \mathrm{p}}^{2}\left(\frac{1}{q_{\mathrm{saf}}} \frac{d q_{\mathrm{saf}}}{d r}\right) \\
& \times \frac{T_{i}}{T_{e}}\left(\frac{1}{N_{e}} \frac{d N_{e}}{d r}+\frac{1}{N_{i}} \frac{d N_{i}}{d r}+\frac{2}{T_{i}} \frac{d T_{i}}{d r}\right) \\
& \hat{U}_{2}^{e i}=3 \rho_{\mathrm{e}, \mathrm{p}}\left(\frac{1}{q_{\mathrm{saf}}} \frac{d q_{\mathrm{saf}}}{d r}\right) \frac{T_{i}}{T_{e}}
\end{aligned}
$$

Here, the electron poloidal gyro-radius and gyro-frequency are defined by, respectively, $\rho_{\mathrm{e}, \mathrm{p}}=v_{\mathrm{th}, \mathrm{e}} / \Omega_{\mathrm{e}, \mathrm{p}}$ and $\Omega_{\mathrm{e}, \mathrm{p}}=e B_{0, \theta} /$ $\left(c M_{e}\right)$. The transport coefficient is given by $\mathcal{L}_{12}=\sum_{r_{a}} p^{2} \pi N_{1}$ $b_{t}^{2} D_{R R}(1,2)$, where $D_{\mathrm{RR}}(1,2)$ is a generalized RechesterRosenbluth coefficient. ${ }^{19}$ In Eq. (1), we only keep the electron flux and source due to the fluctuation spectrum induced by the ions, as shown by the subscript "ei". The electronelectron interaction $\Gamma_{e e}^{V}$ and $U_{e e}^{V}$ is neglected due to the use of the pseudo-thermal ansatz ${ }^{14}$ that makes these terms equal to zero when volume-integrated. However, these terms are locally nonzero, and in order to have a better estimate of their effect, the use of a less restrictive turbulent generalized Balescu-Lenard (gBL) collision operator would be required, one obtained through a self-consistent phenomenological evaluation of the turbulent spectrum.

The first terms in Eqs. (2) and (3) are diagonal terms representing MHD effects, while the others are off-diagonal, purely kinetic terms. The existence of off-diagonal terms in the momentum transport matrix has been predicted theoretically and confirmed experimentally. ${ }^{20}$ The drive in the offdiagonal terms is related to ion and electron density and temperature gradients, as well as the gradient of the safety factor $q_{\text {saf. }}$. The source terms in Eqs. (5) and (6) are proportional to the safety factor gradient, meaning that the momentum source will only play a role when the magnetic shear is large. Differently, in Eq. (4), the safety factor term contains the $T_{i} /$ $T_{e}$ ratio and can change the direction of the momentum flux depending on the electron and ion temperatures and on the magnetic configuration. In conventional scenarios with $q_{\text {saf }}$ monotonically increasing with $r$, a momentum pinch is obtained for $T_{i} / T_{e}<3 / 2$.

The turbulent electric field is derived from the electron momentum balance Eq. (1), where the flux and the source are given in Eqs. (4)-(6). Assuming that for simplicity the ion drift velocity is negligible, $V_{\| i}=0$, and approximating the electron velocity as $V_{\| e} \simeq-j_{\|} /\left(e N_{e}\right)$, Eq. (1) for the passing-electron gives

$$
-\frac{M_{e}}{e^{2} N_{e}} \frac{\partial\left\langle j_{\|}\right\rangle_{r}}{\partial t}+\frac{\left\langle N_{e} E_{t}\right\rangle_{r}}{N_{e}}=\eta_{\text {nea }} j_{\|}-E_{\mathrm{bs}}+E_{\|}^{\mathrm{turb}} .
$$

The RHS of Eq. (7) contains two additional terms, the neoclassical resistive term $\eta_{\text {neo }} j_{\|}$and the neoclassical bootstrap term, $E_{\mathrm{bs}} \propto-d p / d r$, where $p$ is the total equilibrium pressure. The turbulent electric field is then written in the following form:

$$
E_{\|}^{\mathrm{turb}}=\eta_{\mathrm{an}} j_{\|}+\eta_{\times} \frac{d j_{\|}}{d r}-\frac{1}{B_{0} r} \frac{d}{d r}\left[r \eta_{\mathrm{h}} \frac{d}{d r}\left(\frac{j_{\|}}{B_{0}}\right)\right]
$$

in order to make connection to the MHD framework of the mean-field, ${ }^{21,22}$ in which case hyper-resistivity $\eta_{\mathrm{h}}$ is equivalent to the $\alpha$-term in the dynamo theory while anomalous resistivity $\eta_{\text {an }}$ to the $\beta$-term. ${ }^{23}$ In Eq. (8), $B_{0}$ is the total equilibrium magnetic field, and the three transport coefficients (anomalous resistivity, cross-resistivity, and hyper-resistivity) are given by

$$
\eta_{\mathrm{an}} \equiv-\frac{1}{B_{0} r} \frac{d}{d r}\left(\frac{r \Lambda_{2}^{\Gamma}}{N_{e}} \frac{d B_{0}}{d r}\right)+\frac{\Lambda_{1}^{\Gamma}+r\left(d \Lambda_{1}^{\Gamma} / d r\right)}{N_{e} r}-\Lambda_{1}^{U},
$$




$$
\eta_{\times} \equiv-\frac{\Lambda_{2}^{\Gamma}}{N_{e} L_{N_{e}}}+\frac{\Lambda_{1}^{\Gamma}}{N_{e}}+\Lambda_{2}^{U},
$$

and

$$
\eta_{\mathrm{h}} \equiv \Lambda_{2}^{\Gamma} B_{0}^{2} / N_{e}
$$

To shorten the previous equations, we have used the following notations:

$$
\begin{gathered}
\Lambda_{1}^{\Gamma}=\frac{\eta_{e i}}{\rho_{\mathrm{e}, \mathrm{p}}}\left(\hat{\Gamma}_{2}^{e i} \frac{\rho_{\mathrm{e}, \mathrm{p}}}{L_{N_{e}}}-\hat{\Gamma}_{1}^{e i}\right), \Lambda_{2}^{\Gamma}=\eta_{e i} \hat{\Gamma}_{2}^{e i}, \\
\Lambda_{1}^{\mathrm{U}}=\frac{1}{N_{e} \rho_{\mathrm{e}, \mathrm{p}}} \frac{\eta_{e i}}{\rho_{\mathrm{e}, \mathrm{p}}}\left(\hat{U}_{2}^{e i} \frac{\rho_{\mathrm{e}, \mathrm{p}}}{L_{N_{e}}}-\hat{U}_{1}^{e i}\right), \Lambda_{2}^{U}=\frac{1}{N_{e} \rho_{\mathrm{e}, \mathrm{p}}} \eta_{e i} \hat{U}_{2}^{e i}
\end{gathered}
$$

where $L_{N_{e}}^{-1} \equiv\left(1 / N_{e}\right)\left(d N_{e} / d r\right)$, and the transport coefficients are $\eta_{e i}=M_{e} \mathcal{L}_{e i} /\left(e^{2} N_{e}\right)$ and $\mathcal{L}_{e i} / N_{e}=\pi v_{t h, e} q_{s} R_{0} \tilde{b}^{2}$, with $\tilde{b}$ the normalized (to $B_{0}$ ) magnetic perturbation.

\section{TURBULENT TRANSPORT COEFFICIENTS}

The last term in Eq. (8), hyper-resistivity $\eta_{\mathrm{h}}$, originates from the momentum flux. It is proportional to the anomalous viscosity and has a tendency to smooth out the radial gradient of the parallel current density near the axis, which could explain why experimental current profiles remain nonhollow even in the presence of strong bootstrap current. ${ }^{24}$ The magnetic turbulence profiles in this paper are chosen to vanish at the boundary, making the hyper-resistive term helicity conserving. Eq. (8) shows that in a Taylor state, ${ }^{25}$ $j_{\|} / B_{0}=$ constant, the effects of this term are vanishing, and so, we can say that the hyper-resistive term is driven by departures from a Taylor state. This term is also responsible for toroidal field reversal at the plasma edge in pinches. ${ }^{22}$ The remaining two coefficients, the anomalous resistivity Eq. (9) and cross-resistivity Eq. (10), contain both momentum flux and momentum source contributions, a result of the self-consistency of our theory. Hyper-resistivity is always a dissipative term, whereas the cross-resistivity usually generates power from the turbulence. The anomalous term is mostly dissipative, but we show that this can change depending on the temperature and perturbation profile.
In this section, we present the radial dependence of the three turbulent transport coefficients [Eqs. (9)-(11)] as functions of the temperature and turbulence perturbation profile. For the tokamak dimensions, we take $a=71 \mathrm{~cm}$ and $R_{0}$ $=240 \mathrm{~cm}$ and obtain an inverse aspect ratio of $\epsilon \equiv a / R_{0}$ $=0.295$. The electron (and ion) density profile is given by $N_{e}=N_{i}=\left(N_{e, 0}-N_{e, a}\right)\left(1-x^{2}\right)^{\gamma_{N}}+N_{e, a}$, where $x=r / a$, $\gamma_{N}=1.5, N_{e, 0}=2 \times 10^{13} 1 / \mathrm{cm}^{3}$, and $N_{e, a}=2 \times 10^{11} 1 / \mathrm{cm}^{3}$ for all simulations in this paper. The electron and ion temperature profiles are given with the function $T_{e}=T_{i}=\left(T_{e, 0}\right.$ $\left.-T_{e, a}\right)\left(1-x^{\sigma_{T}}\right)^{\gamma_{T}}+T_{e, a}$, where $T_{e, 0}, T_{e, a}, \sigma_{T}$, and $\gamma_{T}$ are parameters to be chosen. We will compare the turbulent coefficients for two L-modes shown in Fig. 1(b), one peaked L-mode (full line) and one broad mode (line with boxes). Additionally, for comparison in the same figure, we show the advanced-like mode (line with circles) that will be studied in Sec. VB. For the peaked L-mode, we take $\gamma_{T}=2.0$ and $\sigma_{T}=2.0$ with boundary values $T_{e, 0}=4.606 \mathrm{keV}$ and $T_{e, a}=0.1 \mathrm{KeV}$. The broad mode is obtained for $\gamma_{T}=2.5$ and $\sigma_{T}=4.5$, with boundary values $T_{e, 0}=2.875 \mathrm{keV}$ and $T_{e, a}=0.09375 \mathrm{keV}$. This mode has a strong gradient in the outer region, and hence, the bootstrap current is expected to be wider compared to the one of the peaked modes. The advanced mode is given by the parameters: $\gamma_{T}=3.0$, $\sigma_{T}=8.0, T_{e, 0}=2.87 \mathrm{keV}$, and $T_{e, a}=0.0878 \mathrm{keV}$. This mode has an even steeper pressure gradient near the edge of the plasma. For all profiles in this section, we will take $T_{e}=T_{i}$, and hence, $p_{e}=p_{i}=p / 2$.

In Ref. 10, simulation was done with magnetic perturbation $\tilde{b} \sim O\left(10^{-4}\right)$ peaked at the center. This level of turbulence is rarely present at the axis where turbulence vanishes due to flat profiles, but it is not uncommon at the edge. While density perturbations are experimentally well measured, the same cannot be said about magnetic perturbations due to the small amplitude and lack of good diagnostics. Experiments with heavy ion probing at the center in the JIPPT-IIU tokamak show a turbulence level of $O\left(10^{-4}\right),{ }^{27}$ which however is not expected to be present at the axis of larger machines. Measurements off-axis in Tore Supra using cross-polarization scattering of microwaves ${ }^{28}$ report similar levels in the L-mode. The level of turbulence we examine in this paper $\left(\tilde{b} \sim O\left(10^{-5}-10^{-4}\right)\right.$ ) provides for the ergodization of the magnetic surface which causes for the electrons streaming along the magnetic field lines to execute a random-walk in a
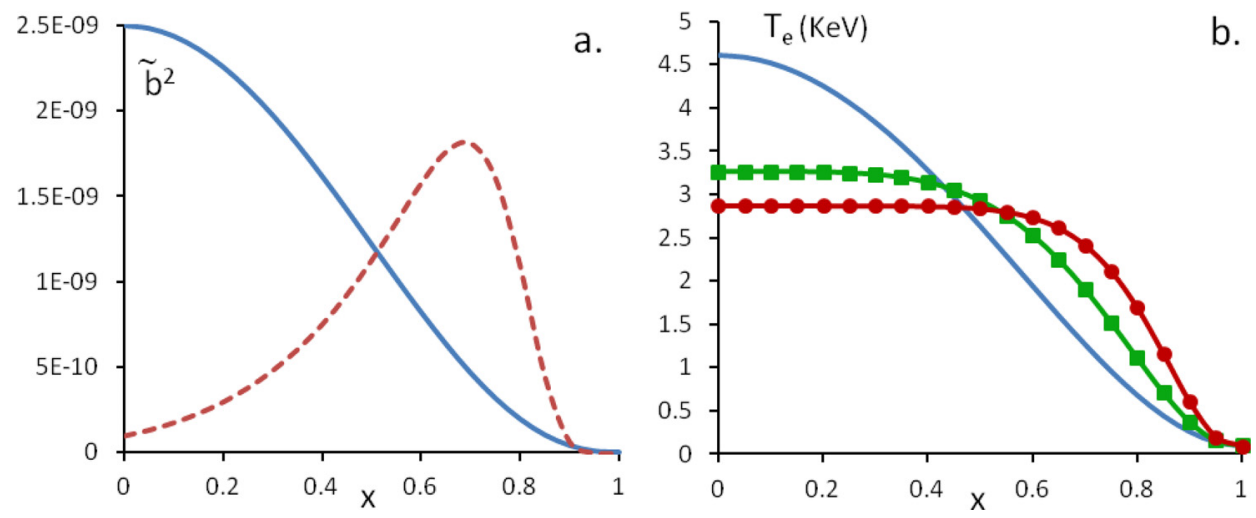

FIG. 1. (a) Profiles of perturbation $\tilde{b}^{2}$ : full line-peaked at the axis and dashed line-peaked off-axis. (b) Electron temperature profiles: full line-peaked L-mode, line with boxes-broad L-mode, and line with circles—advanced scenario. 
stochastic magnetic field. ${ }^{19}$ In contrast to this, the MHD approach to this problem requires small amplitude MHD fluctuations and not necessarily the stochastic field. This is not a limitation of the kinetic theory since some stochastization of the field is present even for significantly lower levels of microturbulence $\tilde{b} \sim O\left(10^{-7}-10^{-6}\right){ }^{29}$

To understand better the effects of the turbulence, we assume the presence of densely packed micro-tearing modes described by two different (intermediate level) perturbation profiles: one peaked at the axis [full line in Fig. 1(a)] given by $\tilde{b}^{2}=0.25 \times 10^{-8}\left(1-x^{2}\right)^{2.5}$ and another for turbulence near the edge with $\tilde{b}^{2}=10^{-10} \times\left(1-x^{10}\right)^{10}(1+x)^{6}$ [dashed line in Fig. 1(a)]. For completeness, we will also show results for stronger turbulence $\tilde{b}^{2}=O\left(10^{-8}\right)$ at the center and weaker $\tilde{b}^{2} \lesssim O\left(10^{-10}\right)$ everywhere in the plasma. For a more accurate analysis, in future works, we will compare our results with turbulence profiles obtained from more consistent numerical codes. All perturbations in this work are taken to be zero at the plasma edge, leading to a helicityconserving hyper-resistivity. Since, at the moment, we are more interested in the core plasma, this assumption is justified, even though it neglects the proper treatment of edge current diffusion and possibly the effects of turbulence on impurity transport. All turbulent coefficients are further normalized with $\bar{\eta}_{\mathrm{cl}}$, the cylindrical cross-section average of the classical resistivity $\eta_{\mathrm{cl}}=4.77 e^{2} Z_{\mathrm{eff}} \ln \Lambda /\left[M_{e} v_{\mathrm{th}, e}^{3}\right]$, where the Coulomb logarithm and the ion charge are $\ln \Lambda=17$ and $Z_{\text {eff }}$ $=1$, respectively. To avoid the singularity of the neoclassical resistivity $\eta_{\text {neo }}=\eta_{\mathrm{cl}} /\left(1-1.95 \sqrt{r / R_{0}}\right)$, we will simply take dimensional hyper-resistive coefficient $\hat{\eta}_{\mathrm{h}}=\eta_{\mathrm{h}} /\left(a^{2} B_{z, 0}^{2} \bar{\eta}_{\mathrm{cl}}\right)$, cross coefficient $\hat{\eta}_{\mathrm{X}}=\eta_{\mathrm{X}} /\left(a \bar{\eta}_{\mathrm{cl}}\right)$, and the anomalous coefficient $\hat{\eta}_{\mathrm{an}}=\eta_{\mathrm{an}} / \bar{\eta}_{\mathrm{cl}}$ are plotted in Figs. 2(a)-2(c), respectively, for two different temperature and turbulent profiles. In Fig. 2, the full line represents the peaked mode with axis turbulence, the dashed line represents the peaked mode with edge turbulence, and the line with boxes represents the broad mode with axis turbulence. All coefficients becomes zero at the edge of the plasma due to the adopted shape of the magnetic perturbation, while in the inner region, they are all sensitive to the equilibrium $(N, T)$ and turbulence $\left(\tilde{b}^{2}\right)$ profiles. Hyper-resistivity is proportional to $\tilde{b}^{2}$ [see Eq. (11)] and takes on the shape of the $\eta_{\text {neo }}=\eta_{\mathrm{cl}}$ since this issue is not essential to our study. The non-

perturbation, while the shapes of the other two do not change in a significant way when switching from the axis to edge turbulence. However, the values of all three parameters in this situation decrease near the axis but significantly increase in the outer region. This is due to the alignment of the turbulence peak with the temperature gradient. The coefficients are also generally larger for a broader mode.

While the hyper- and the cross-resistive contributions are positive everywhere in the plasma, the anomalous resistivity switches the sign from positive to negative when moving towards large $x$, depending on equilibrium thermodynamic and magnetic $\left(q_{\text {saf }}\right)$ profiles, as Eqs. (4)-(6) show. This term is on average larger than the other two, but it is still significantly smaller than the classical resistivity. In Eq. (9), we have written $\hat{\eta}_{\text {an }}$ as a sum of three terms, of which the second one is shown to be dominant. ${ }^{10}$ This coefficient has a removable singularity at the axis [see Eq. (9)] that disappears when Ohm's law is iteratively solved due to the readjustment of the magnetic profile. The existence of the negative anomalous resistivity region has already been postulated in several works using the MHD approach. ${ }^{9}$ In Sec. V B, we show that in advanced scenarios, anomalous resistivity can generate power instead of dissipating it, and this to the best of our knowledge is a novel result.

\section{OHM'S LAW AND POWER BALANCE}

The steady state version of Eq. (7) can be written as $E_{\|}^{0}=\eta_{\text {neo }}\left(j_{\|}-j_{\text {bs }}\right)+E_{\|}^{\text {turb }}$ or, using Eq. (8),

$$
\begin{aligned}
E_{\|}^{0}= & -\eta_{\text {neo }} j_{\mathrm{bs}}+\left(\eta_{\text {neo }}+\eta_{\mathrm{an}}\right) j_{\|}+\eta_{\times} \frac{d j_{\|}}{d r} \\
& -\frac{1}{B_{0} r} \frac{d}{d r}\left[r \eta_{h} \frac{d}{d r}\left(\frac{j_{\|}}{B_{0}}\right)\right],
\end{aligned}
$$

where for the neoclassical bootstrap current we adopt: ${ }^{26,30-33}$

$$
\begin{aligned}
j_{\mathrm{bs}}= & -c F_{13}\left(\frac{r}{R}\right)^{1 / 2} \frac{n_{e}\left(T_{e}+T_{i}\right)}{B_{\theta}}\left(\frac{1}{n_{e}} \frac{d n_{e}}{d r}\right)-c\left(\frac{r}{R}\right)^{1 / 2} \frac{n_{e} T_{e}}{B_{\theta}} \\
& \times\left[-\left(\frac{3}{2} F_{13}-F_{23}\right)\left(\frac{1}{T_{e}} \frac{d T_{e}}{d r}\right)\right. \\
& \left.-\left(\frac{3}{2}-y\right) F_{13} \frac{T_{i}}{T_{e}}\left(\frac{1}{T_{i}} \frac{d T_{i}}{d r}\right)\right] .
\end{aligned}
$$

Here
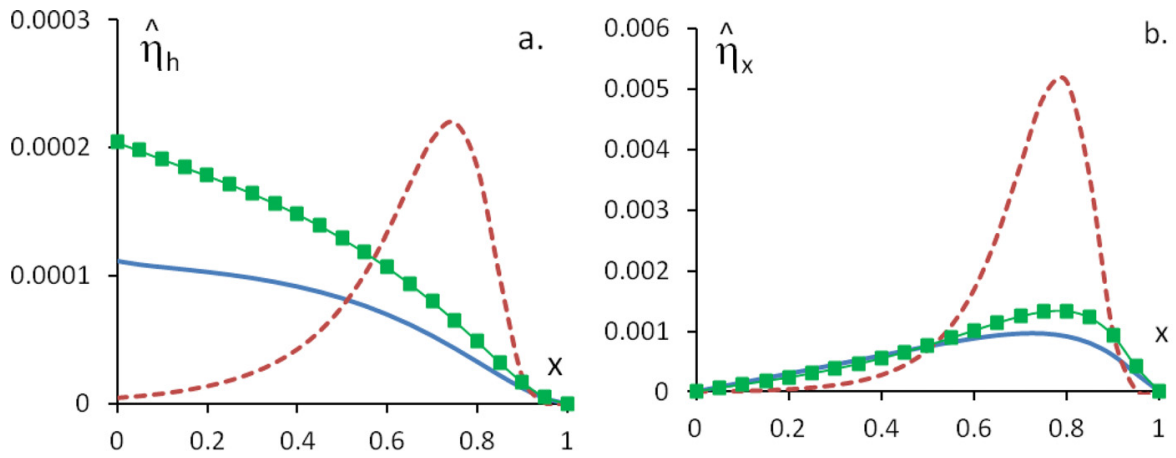

b.

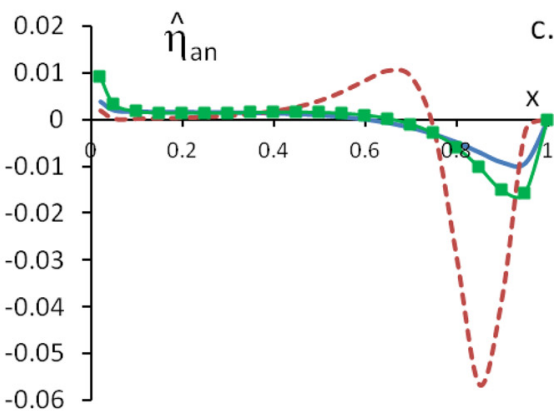

FIG. 2. Normalized turbulent coefficients: (a) hyper-resistivity, (b) cross-resistivity, and (c) anomalous resistivity. The full line indicates the peaked mode and axis turbulence, the dashed line indicates the peaked mode and edge turbulence, and the line with boxes indicates the broad mode and axis turbulence. 


$$
\begin{aligned}
F_{m 3} & =\frac{K_{m 3}}{\left[1+a_{m 3} \nu_{e *}^{1 / 2}+b_{m 3} \nu_{e *}\right]\left[1+c_{m 3} \nu_{e *}(r / R)^{3 / 2}\right]}, \\
m & =1,2, \\
y & =\frac{1.31\left(1+1.65 \nu_{i *}^{1 / 2}\right)}{1+0.862 \nu_{i *}^{1 / 2}}, \\
\nu_{j *} & =\frac{B_{z} R^{3 / 2}}{\tau_{j} r^{1 / 2} B_{\theta}\left(T_{j} / m_{j}\right)^{1 / 2}}, \quad j=e, i,
\end{aligned}
$$

the classical e-i and $\mathrm{i}-\mathrm{i}$ collision times are $\tau_{e}=3 m_{e}^{1 / 2} T_{e}^{3 / 2} /$ $\left[4(2 \pi)^{1 / 2} \ln \Lambda_{e} n_{e} e^{4} Z_{\mathrm{eff}}\right], \tau_{i}=3 m_{i}^{1 / 2} T_{i}^{3 / 2} /\left[4 \pi^{1 / 2} \ln \Lambda_{i} n_{i} e^{4}\right], \quad$ and for $Z_{\mathrm{eff}}=1$, we have $K_{13}=2.30, K_{23}=4.19, a_{13}=1.02$, $a_{23}=0.57, b_{13}=0.75, b_{23}=0.38, c_{13}=1.07$, and $c_{23}=0.61{ }^{32}$ The frequency $\nu *$ is a measure of the collisionality of the plasma and is given by the ratio of the effective collision frequency to the bounce frequency. There are three main collisionality regimes: $\nu_{*} \ll 1$, banana (collisionless) regime; $1 \leq \nu_{*} \leq 1 / \epsilon^{3 / 2}$, plateau regime; and $\nu_{*} \gg 1$, Pfirsich-Schlüter (collisional) regime. Here, we obtain an average value of $\nu_{e^{*}} \approx 0.05$, which falls in the banana regime, as is necessary for maintaining a bootstrap current. Since the poloidal field $B_{\theta}$ appears in the denominator of the expression for the bootstrap current Eq. (15) and is connected to the parallel current density via Ampere's law, $(4 \pi / c) j_{\|} \simeq(1 / r)(\partial / \partial r) r B_{\theta}$, Eq. (14) is a nonlinear integro-differential equation and is therefore solved iteratively.

Ohm's law [Eq. (14)] represents a steady-state balance between charged particle momentum gain and momentum loss expressed through the parallel current density profile. ${ }^{10}$ Solutions of this equation can explain whether or not the turbulent contributions of Ohm's law can provide the necessary current diffusion towards the axis in operations with a large bootstrap component. ${ }^{3,4,33}$ Our work shows that all three terms play a significant role in shaping the current and safety factor profile in the presence of magnetic turbulence and hence must be retained in the analysis. A complete study of the effects of these terms on the evolution of plasma equilibrium should include a transport code which couples the turbulent Ohm's law to the time dependent equations for the temperature and density. Additionally, the turbulence profile and intensity would also change self-consistently. Here, we will concentrate on solving Eq. (14) for a steady-state current profile in a (cylindrical) tokamak with fixed pressure equilibrium profiles and for a fixed (in time) level of magnetic turbulence.

We can further investigate each resistive term using the following power balance equation:

$$
\begin{aligned}
& \underbrace{\int_{0}^{a} d r r j_{\|} E_{\|}^{0}}_{\mathcal{T}_{\mathrm{E}}}+a\left\{\eta_{h} \frac{j_{\|}}{B_{0}} \frac{d}{d r}\left[\frac{j_{\|}}{B_{0}}\right]-\frac{1}{2} \eta_{\times} j_{\|}^{2}\right\}_{r=a} \\
& =\underbrace{\int_{0}^{a} d r\left[\eta_{\text {neo }}+\eta_{\text {an }}\right] r j_{\|}^{2}}_{\mathcal{T}_{\text {neo }}+\mathcal{T}_{\text {an }}}+\underbrace{\int_{0}^{a} d r r \eta_{h}\left[\frac{d}{d r}\left(\frac{j_{\|}}{B_{0}(r)}\right)\right]^{2}}_{\mathcal{T}_{\mathrm{h}}} \\
& +\underbrace{\int_{0}^{a} d r r j_{\|}\left[-\eta_{\mathrm{nea}} j_{\mathrm{bs}}-\frac{1}{2 r} \frac{d\left[r \eta_{\times}\right]}{d r} j_{\|}\right]}_{\mathcal{T}_{\mathrm{BS}}+\mathcal{T}_{\times}},
\end{aligned}
$$

obtained by multiplying Eq. (14) with $r j_{\|}$and then integrating from 0 to $a$, according to the procedure adopted in Refs. 4 and 10. The first term on the LHS is positive and describes the externally injected power by the induced electric field $E_{\|}^{0}$. The second term is the power injected from the boundary surface $(r=a)$ of the plasma, which in this case is equal to 0 due to the mentioned conservation of helicity $\left(j_{\|}(a) \simeq 0\right)$. The first term on the RHS shows the internal dissipation due to neoclassical resistivity and dissipation (or generation) due to anomalous effects. Fig. 2(c) shows that the anomalous resistivity is negative (and generates current) in the outer region of the plasma. However, the current density is generally small in the same region so that in most common cases the overall effect of the anomalous term is dissipation. However, this can change when there is an alignment of the region of negative anomalous resistivity with a strong plasma pressure gradient (see Sec. VB). Most of the power generated internally in all operations comes from the diffusion-driven (bootstrap) electromotive force, reinforced by the turbulent contribution from $\eta_{\times}$. It is shown in Sec. V C that the total bootstrap term $\mathcal{T}_{\text {BS }}+\mathcal{T}_{\times}$could balance the dissipative terms on the RHS, eliminating the need of externally supplied power. Finally, the second term on the RHS represents the additional power (always) dissipated by the hyperresistive current diffusion.

\section{NUMERICAL SOLUTION OF THE CURRENT EQUATION}

When numerically solving Eq. (14), we will use $x \equiv r / a$ as a radial variable and put the relevant quantities in dimensionless form (marked with an over-hat): $\hat{j}_{\|}=4 \pi a j_{\|} /\left(c B_{z, 0}\right)$, $\hat{E}_{\|}^{0}=4 \pi a E_{\|}^{0} /\left(\bar{\eta}_{\mathrm{cl}} c B_{z, 0}\right), \hat{P}=P / B_{z, 0}^{2}, \hat{B}=B / B_{z, 0}, \hat{\eta}_{\text {neo }}=\eta_{\text {neo }} /$ $\bar{\eta}_{\mathrm{cl}}, \hat{\eta}_{\mathrm{h}}=\eta_{\mathrm{h}} /\left(a^{2} B_{z, 0}^{2} \bar{\eta}_{\mathrm{cl}}\right)$, and $\hat{\eta}_{\times}=\eta_{\times} /\left(a \bar{\eta}_{\mathrm{cl}}\right), \hat{\eta}_{\mathrm{an}}=\eta_{\mathrm{an}} / \bar{\eta}_{\mathrm{cl}}$, where $\bar{\eta}_{\mathrm{cl}}$ is the volume averaged classical resistivity and $B_{z, 0}=4 \times 10^{4} \mathrm{G}$ is the toroidal field. Then, the final form of the parallel current equation is

$$
\begin{gathered}
\hat{D}_{2}(x) \frac{d^{2} \hat{j}_{\|}(x)}{d x^{2}}+\hat{D}_{1}(x) \frac{d \hat{j}_{\|}(x)}{d x}+\hat{D}_{0}(x) \hat{j}_{\|}(x) \\
=\frac{\hat{D}_{-1}(x)}{\int^{x} d x^{\prime} x^{\prime} \hat{j}_{\|}\left(x^{\prime}\right)}+\hat{E}_{\|}^{0}(x),
\end{gathered}
$$

with

$$
\begin{aligned}
\hat{D}_{2}\left(x ; \eta_{\mathrm{h}}\right)= & -\frac{\hat{\eta}_{\mathrm{h}}}{\hat{B}_{0}^{2}}, \hat{D}_{1}\left(x ; \eta_{\mathrm{h}}, \eta_{\times}\right)=\hat{\eta}_{\times}-\frac{1}{\hat{B}_{0, z}^{2}} \frac{1}{x} \frac{d\left(x \hat{\eta}_{\mathrm{h}}\right)}{d x}, \\
\hat{D}_{0}\left(x ; \eta_{\mathrm{neo}}, \eta_{\mathrm{an}}\right)= & \hat{\eta}_{\mathrm{neo}}+\hat{\eta}_{\mathrm{an}}, \\
\hat{D}_{-1}\left(x ; \eta_{\mathrm{neo}}\right)= & -4 \pi \hat{\eta}_{\text {neo }}\left(\frac{a}{R_{0}}\right)^{1 / 2} x^{3 / 2} \times\left\{F_{13} \frac{n_{e}\left[T_{e}+T_{i}\right]}{B_{z, 0}^{2}}\right. \\
& \times\left(\frac{1}{n_{e}} \frac{d n_{e}}{d x}\right)+\frac{n_{e} T_{e}}{B_{z, 0}^{2}} \times\left[-\left(\frac{3}{2} F_{13}-F_{23}\right)\right. \\
& \left.\left.\times\left(\frac{1}{T_{e}} \frac{d T_{e}}{d x}\right)-\left(\frac{3}{2}-y\right) F_{13} \frac{T_{i}}{T_{e}}\left(\frac{1}{T_{i}} \frac{d T_{i}}{d x}\right)\right]\right\},
\end{aligned}
$$


where $\hat{D}_{1}$ and $\hat{D}_{0}$ were expanded to the lowest order term of the small parameter $\epsilon \sim B_{0, p} / B_{0, z} \sim a / R_{0}$. We would like to correct a typographical error in Ref. 10 where in Eq. (39), the derivatives should be with respect to $x$, not $r$.

A numerical code, Turbulent Ohm's Law Solver (abbr. TOLS), iteratively solves the nonlinear Eq. (17) by taking the Ohmic + Bootstrap current as the initial solution and recalculating the fields, safety factor, coefficients, and the current when turbulence is added, until a converged solution is achieved. The effect of the turbulent terms limited to a peaked L-mode profile has been studied in Ref. 10. To better understand the importance of each term, in this paper, we use several different scenarios and two different turbulence profiles. For simplicity sake, we take the ion and electron temperatures to be equal in all scenarios, except for the fully bootstrapped tokamak in Sec. VC, which includes an electron transport barrier. As previously stated, we approximate $\hat{\eta}_{\text {neo }} \simeq \hat{\eta}_{\mathrm{cl}}$ and assume the boundary condition $\hat{j}_{\|}(x=1)=0$ in all cases, while the additional condition $\hat{j}_{\|}^{\prime}(x=0)=0$ is applied when the hyper-resistivity is taken into account. The normalized inductive field is $\hat{E}_{\|}^{0}=7.0625 \times 10^{-3}$. All parameters are chosen so that the stored energy is equal in all scenarios, while the current inside the plasma (Ohmic + Bootstrap) is 700KA.

\section{A. L-mode type profiles}

In this section, we will consider the two temperature profiles from Fig. 1(b), the peaked mode represented by the full line and the broad mode by the line with boxes. In the peaked L-mode temperature profile, the bootstrap current constitutes $14.6 \%$ of the initial current. In Ref. 10, it was shown that the hyper-resistivity, although the smallest among the coefficients, plays an important role near the axis by diffusing the current density toward the center and hence flattening the current profile. This effect is essential in dealing with the hollowness induced by the bootstrap current in advanced scenarios (see Secs. V B and V C). Checking first the effect of each single turbulent term in isolation, using the on-axis turbulence profile of Fig. 1(a), we find that the hyper-resistivity reduces the current by $1.2 \%$. The anomalous resistivity reduces the current in the central region of the plasma but also increases it in the outer region due to the negative value of $\hat{\eta}_{\text {an }}$, with the overall effect being reduced by roughly $2.5 \%$. The crossresistivity on the other hand increases the current everywhere in the plasma with total amplification by some 3\%. We explicitly note that the increases/reductions of the current due to the turbulent terms do not simply add but combine according to a complicated differential equation.

When all turbulent coefficients are included, we obtain the current profiles in Fig. 3(a), where the full line indicates Ohmic + Bootstrap current only, the line with boxes indicates axis turbulence, the dashed line indicates edge turbulence, and the line with circles indicates strong turbulence $O\left(10^{-4}\right)$ on axis. For the moderate level of turbulence, the final effect of all turbulent terms is reduction of the current by $1 \%$, which means that the total current before and after inclusion of the turbulence is for all practical purposes unaltered. However, we see from Fig. 3(a) (line with boxes) that the turbulence internally redistributes about $5.5 \%$ of the total current or in absolute values, $2.43 \mathrm{~A} / \mathrm{cm}^{2}$. In this scenario, two terms generate power internally (given in relative values), $\mathcal{T}_{\times}=3.2 \%$ and $\mathcal{T}_{\mathrm{BS}}=15.8 \%$, with the rest generated inductively, while the other terms dissipate the power as follows: $\mathcal{T}_{\mathrm{h}}=1.1 \%, \mathcal{T}_{\text {an }}=2.6 \%$, and the majority by the neoclassical term. When the turbulence is peaked off-axis, the current change around the axis is negligible, but there is some current change in the outer region [Fig. 3(a)-dashed line], and due to the competing effects of the anomalous and cross-resistivity, there is a small current reduction of $1 \%$. When turbulence is strong at the center $\left(O\left(10^{-4}\right)\right.$ shown as the line with circles), there is still a small reduction of $4 \%$ but a significant current redistribution, while for $\tilde{b}<10^{-5}$ everywhere in the plasma, the effects become negligible (order $0.1 \%$ of the total current). For the intermediate level of turbulence, the redistribution of the current is enough to cause the $q_{\text {saf }}$ profile to raise [see Fig. 3(b)]. This is important in discharges with $q_{s a f}<1$ on axis, where increasing the value to $q_{\text {saf }}>1$ will prevent a sawtooth crash. A paper on one such case from ASDEX Upgrade is in preparation.

Several works have considered the existence of sawtoothfree hybrid discharges and credited the stability of the stationary state to several different phenomena: hyper-resistivity, ${ }^{34}$ rotating island driving drift current, ${ }^{35}$ critical poloidal current density, ${ }^{36} 3 / 2$ tearing mode, ${ }^{37}$ fishbone activities, ${ }^{38,39}$ and the
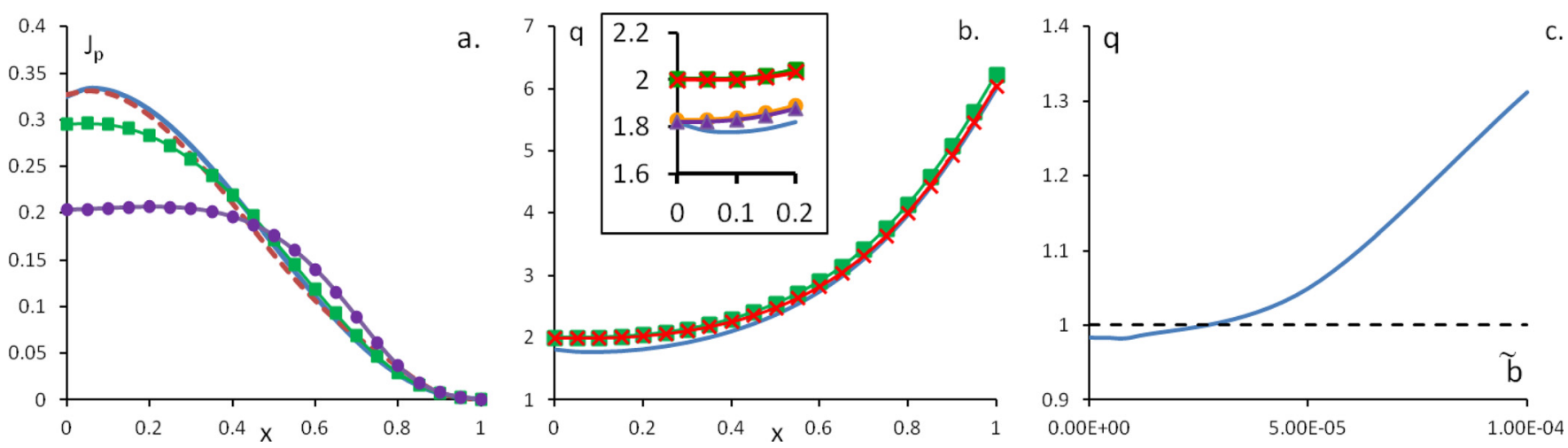

FIG. 3. (a) Parallel current of the peaked L-mode. The full line indicates Ohmic + Bootstrap current only, the line with boxes indicates axis turbulence, the dashed line indicates edge turbulence, and the line with circles indicates $O\left(10^{-4}\right)$ axis turbulence. (b) Safety factor of the peaked L-mode with axis turbulence: full line-Ohmic + Bootstrap current only, line with circles-added hyper-resistivity, triangles—hyper + cross-resistivity, line with boxes-hyper + anomalous resistivity, and line with crosses—all coefficients. (c) Safety factor at the axis vs. $\tilde{b}$. 
exact effect of which on the safety factor is yet to be clarified. In a simulation using the M3D-C1 (3D resistive MHD) code, ${ }^{40}$ the $q$-profile is raised via generation of an interchange mode at the axis that adjusts the loop voltage through dynamo. Steady-state turbulence, which to some degree is always present in tokamaks, can co-exist with other modes and hence, not contradicting any of the previously mentioned works can explain why there is a stationary non-sawtooting state over resistive time scales. In Fig. 3(b), we show how the safety factor changes when various combinations of turbulent coefficients act together. The full line represents the slightly reversed shear obtained from Ohmic and bootstrap current, while the upper two profiles (lines with boxes and triangles) show $q_{\text {saf }}$ when anomalous resistivity is included. The rest (lines with circles and crosses) are without anomalous resistivity and do not significantly change the $q$-profile. We conclude that the increase in the value of $q_{\text {saf }}$ is caused by current reduction by the anomalous term at the axis, making this term a possible candidate for the suppression of sawtooth crashes. In Fig. 3(c), we show how the $q$-factor on the axis changes with the level of turbulence $\tilde{b}$ for a $1.15 M A$ discharge with $q_{0}=0.983$ on the axis. We note that a certain level (threshold) of turbulence is required to raise the profile above $q_{0}=1$. For $\tilde{b}<10^{-5}$, the value of $q_{\text {saf }}$ is diminishing slightly with $\tilde{b}$ before it starts to grow. This is due to the hyper-resistivity which always flattens the surplus bootstrap current at the axis. For larger $\tilde{b}$, the effects of anomalous resistivity start to dominate and the safety factor is increased. From this discussion, it becomes obvious that the turbulent threshold for stabilization of the sawtooth is related to the thermodynamic profiles and the bootstrap current size and peak location.

For the broad L-mode represented the line with boxes in Fig. 1(b), the bootstrap current constitutes $19.8 \%$ of the total current and is radially wider compared to the one of the peaked modes, thus giving the current profile represented as the full line in Fig. 4(a). The shear is reversed over a wide region, which has some advantages concerning confinement and MHD stability (see Sec. V B for details). When the turbulence is peaked on axis, the final current after redistribution is represented with the line with boxes in Fig. 4(a), while for turbulence peaked at the edge, the current is significantly modified [Fig. 4(a), dashed line] due to the alignment of the turbulence peak with strong temperature gradients. The power is generated as $\mathcal{T}_{\times}=2.8 \%$ and $\mathcal{T}_{\text {BS }}=20.3 \%$, with majority still coming from the inductive current. The anomalous resistivity still reduces the total current but generates a bump near the edge which cannot be diffused towards the center. The total current redistribution is about $5 \%$, and it goes up to $16.8 \%$ for $\tilde{b}=O\left(10^{-4}\right)$ (line with circles), while for $\tilde{b}=O\left(10^{-5}\right)$, it is below $1 \%$.

\section{B. Advanced-like scenario}

Generally speaking, any scenario which has significantly improved confinement and MHD stability over the standard $\mathrm{H}$-mode can be referred to as an advanced scenario. These modes have a broad current profile and larger bootstrap current compared to the conventional (peaked) modes. A broad current affects negatively the confinement since the confinement time grows with the current peaking. ${ }^{41}$ Additionally, MHD stability suffers since both the external kink mode ${ }^{42}$ and ballooning mode are more unstable for broader currents. ${ }^{41}$ However, the presence of a transport barrier along with the large bootstrap current in advanced scenarios creates a reversed shear in a broad region, which makes the ballooning modes and the neoclassical tearing mode stable. The kink modes can be stabilized, for relatively high $\beta_{N}$ using a conducting wall. ${ }^{43}$

In this section, we will use the temperature profile is represented by the line with circles in Fig. 1 to simulate an advanced scenario. This mode has a broad enough profile with a bootstrap current peaked near the edge and constituting $25 \%$ of the total current. When the turbulence is peaked on axis, there is negligible current generated from it. The profile remains broad and becomes less hollow at the axis [Fig. 5(a), line with boxes]. More importantly, the shear is still reversed over a wide region [Fig. 5(b), line with boxes], making this in a way the ideal scenario. The bootstrap current generates $\mathcal{T}_{\text {BS }}=24.9 \%$ of the power and cross-resistivity $\mathcal{T}_{\times}=2.8 \%$, while the anomalous and hyper-resistivity dissipate below $1 \%$ of the power. When speaking of anomalous resistivity, the case of an advanced scenario with edge turbulence (dashed lines in Fig. 5) deserves special attention. In this scenario, the anomalous resistivity generates $\mathcal{T}_{\text {an }}=5.6 \%$ of the power, which is similar to the cross-resistivity with $\mathcal{T}_{\times}=5.8 \%$ and about $1 / 4$ of the bootstrap current with $\mathcal{T}_{\text {BS }}=23.8 \%$. By turning on and off each turbulent coefficient, we conclude that the bump on the current near the edge is caused by the anomalous resistivity. This occurs due to the alignment of the region of negative anomalous resistivity with the temperature gradient and turbulence peak. This edge current does not
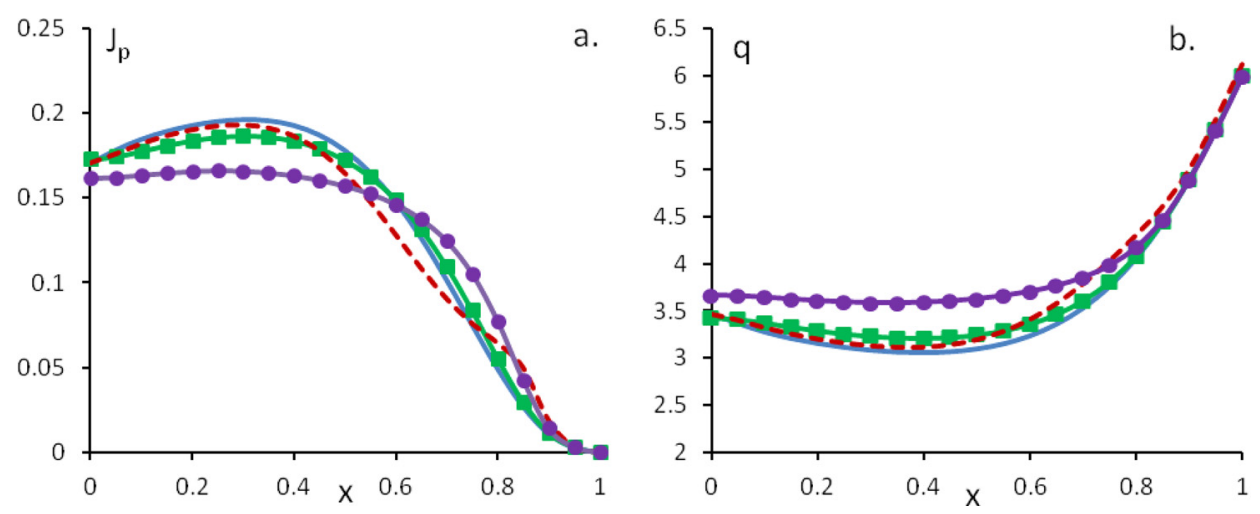

FIG. 4. (a) Parallel current and (b) safety factor of the broad L-mode. The full line indicates Ohmic + Bootstrap current only, the line with boxes indicates axis turbulence, the dashed line indicates edge turbulence, and the line with circles indicates $O\left(10^{-4}\right)$ axis turbulence. 

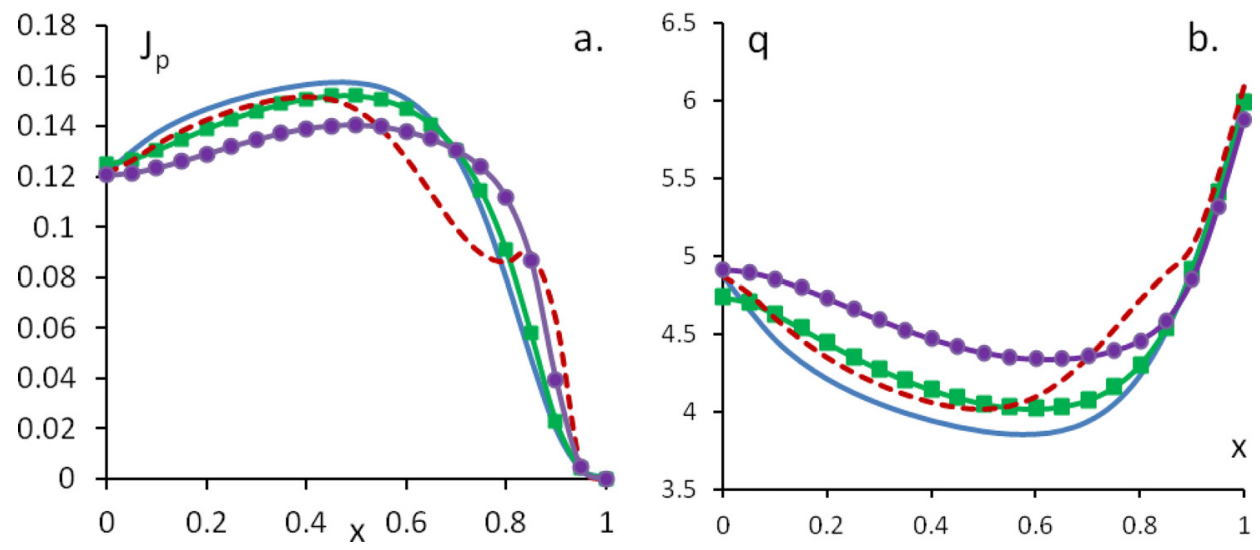

FIG. 5. (a) Parallel current and (b) safety factor of advanced scenarios. The full line indicates Ohmic + Bootstrap current only, the line with boxes indicates added axis turbulence, the dashed line indicates edge turbulence, and line with circles indicates $O\left(10^{-4}\right)$ axis turbulence.

diffuse towards the center, giving large redistribution of the current $(16.6 \%)$ and visibly changing the $q$-profile [dashed line in Fig. 5(b)]. Cross-resistivity increases the total current by about $5 \%$, but this effect is matched by the anomalous resistivity current reduction. Even for stronger turbulence $O\left(10^{-4}\right)$ (line with circles), the total current is only increased by $2 \%$. Since experimentally the exact current profile is difficult to measure especially near the axis, considering only the total parallel current, most experiments would report negligible effects from turbulence.

\section{Fully bootstrapped tokamak}

These scenarios are usually related to internal transport barriers, where the current is generated by the strong pressure gradient, and the confinement is maintained by power generation by the bootstrap current through an internal loop. The alignment of the bootstrap current with the internal transport barrier is hence essential for the maintenance of the regime. In this section, we use the temperature profile (in shape, not the absolute value) from a steady state, fully bootstrapped discharge in the TCV tokamak. ${ }^{7,44}$ The experimental data are fitted with the formula $T_{e}=0.1 / \sqrt{x^{5.5}+0.02^{2}} \mathrm{keV}$ to obtain the temperature profile in Fig. 6(a). For the ion temperature, we use the same formula as in Secs. VA and VB with $\gamma_{T}=2.0, \sigma_{T}=2.0$ and boundary values $T_{e, a}=0.25 \mathrm{keV}$ and $T_{e, 0}=1.25 \mathrm{keV}$. The density is the same as every simulation so far. With this equilibrium, the current is generated $100 \%$ via the bootstrap effect $\left(E_{\|}^{0}=0\right)$. The full line in Fig. 6(b) shows the bootstrap current as calculated by our model (compared to Fig. 4 of Ref. 44). The broadness of the profile is smaller compared to the advanced scenario since the transport barrier is near the axis. Our code is a steady-state code, and so, it cannot show how the internal current-temperature loop maintains the confinement. For a detailed description of how the steady state is achieved, we refer the reader to Ref. 7.

In this scenario, it is not unusual to have a high level of perturbations around the axis since the temperature gradient is strong in this area. The plots with the line with boxes and dashed line in Fig. 6(b) are for the turbulence profiles chosen so far using hyper-resistivity and cross-resistivity, while the line with circles is for stronger turbulence $\left(O\left(10^{-4}\right)\right)$. We note that hyper-resistivity is still effective in diffusing the current towards the center. The dissipation by hyperresistivity is significant in this case $\left(\mathcal{T}_{\mathrm{H}}=9 \%\right)$, and the total current reduction is about $20 \%$, while when cross-resistivity is added, additional current is generated, but the total current is still smaller by $10 \%$ than the initial bootstrap current. The cross-resistivity generates about $\mathcal{T}_{\times}=18 \%$ of the power, while the rest $\mathcal{T}_{\text {BS }}=82 \%$ comes from the bootstrap current. With the anomalous coefficient present, however, we could not find a steady state solution of the equation. While the hyper-resistivity and cross-resistivity "push" the current toward the axis with some generation in the outer region by the cross-resistivity, the anomalous resistivity, which in this scenario is unusually high, significantly reduces the current at the axis, thus affecting the magnetic field and safety factor stability. Whether the presence of anomalous resistivity is
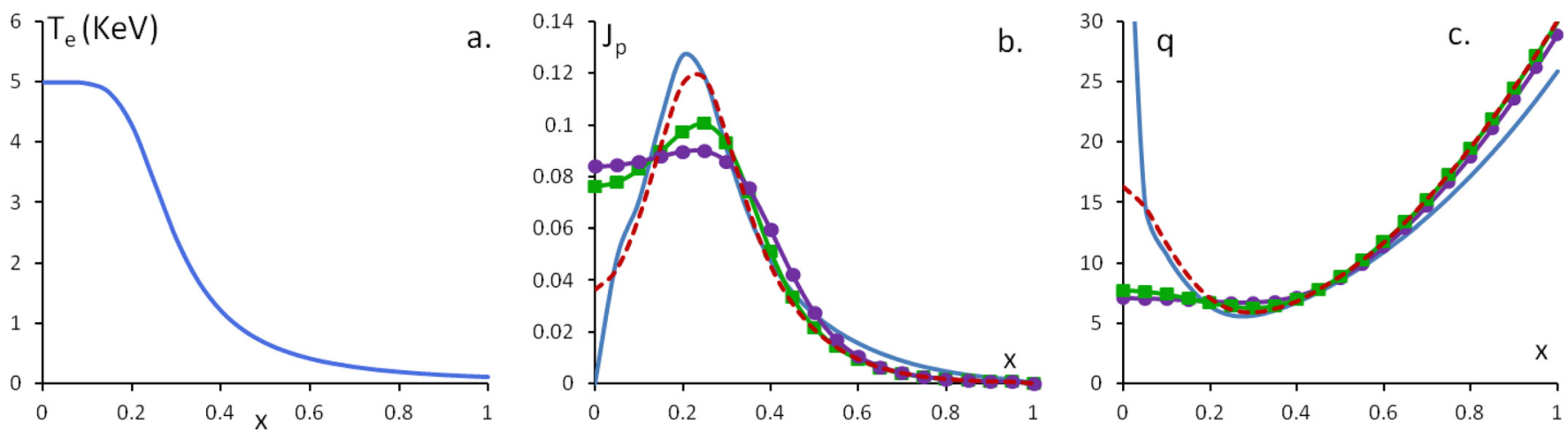

FIG. 6. Fully bootstrapped tokamak: (a) temperature profile and (b) current profile: full line-the bootstrap current, line with boxes—added axis turbulence, dashed line-edge turbulence, and line with circles- $O\left(10^{-4}\right)$ axis turbulence. (c) Safety factor. 
really detrimental to the stability of the profiles is something that should be studied in coordination with a more complete transport code.

The other two coefficients do exactly what is necessary to deal with the hollow current profile, i.e., they diffuse the current towards the center and flatten the profile. However, this results in a change in the safety factor from strongly reversed in the region with the transport barrier to a safety factor which is only slightly reversed [Fig. 6(c)] over a broad region. This means that due to turbulence, some of the mentioned confinement advantages of the reversed shear are lost. It should be mentioned here that obtaining a higher bootstrap fraction and obtaining higher confinement are two different goals of the tokamak development, and today's tokamaks are not designed for optimal bootstrap effects but for confinement and MHD stability. When the turbulence is peaked off-axis, the profiles remain similar to those given in Fig. 6, and hence, the same conclusions apply in that case too. When the fluctuation level is of $O\left(10^{-5}\right)$, the turbulence can still redistribute some current at the axis; however, this effect is smaller and the current profile remains hollow. In this case, additional effects like potato orbits should be taken into account.

\section{SUMMARY AND CONCLUSIONS}

Numerically solving a turbulent version of Ohm's law Eq. (14), we have extended the study presented in Ref. 10 to include advanced plasma regimes, broad L-modes, and fully bootstrapped tokamaks, modeling the magnetic turbulence with two different spectra-one peaked on-axis and one offaxis. Three turbulent terms are present in the generalized Ohm's law: the hyper-resistive term, the anomalous resistivity, and the "cross" resistive term, with the latter being proportional to the derivative of the current density. The anomalous resistivity is the largest coefficient, although all three of them are much smaller than the neoclassical resistivity. Despite this, we find that the turbulent terms have an important impact on the equilibrium current density and safety factor profiles and thus should be retained in Ohm's law. The results of our study better elucidate the impact that each turbulent contribution has on shaping the current and safety factor profiles, as well as on the power generation inside the plasma. Aside from the magnitude of the turbulent perturbations, a key feature characterizing various scenarios is the degree of alignment between the temperature gradient and the turbulence peak.

In L-modes and advanced scenarios, the well-known dissipative hyper-resistive term reduces the total current while diffusing the bootstrap current toward the plasma center. When both the bootstrap current and turbulence profile are peaked in the outer region, the hyper-resistive diffusion is less efficient. The hyper-resistivity is most important in scenarios with high bootstrap current peaked near the axis, such as fully bootstrapped tokamak scenarios, where it plays a crucial role in maintaining a non-hollow current profile. The anomalous resistivity leads to a significant reduction of the current density in the central part of the plasma and to a small increase in the outer region where it has a negative value due to the combined effect of the thermodynamic and magnetic equilibrium profiles. Although usually dissipative, this term can generate power when there is an alignment of its negative peak with the temperature gradient and the turbulent spectrum, which is the case in some advanced scenarios. The cross-resistive term, contrary to the anomalous resistive term, always amplifies the current by roughly $20 \%-25 \%$ of the bootstrap current, depending on the scenario. This term is most commonly generating power, except in rare cases when there is a strong current peak coinciding with the region of the negative slope of the cross-resistivity [see Eq. (16)].

While the competing effects of the three turbulent coefficients on the integral parallel current inside the plasma cancel out in most cases, the redistribution of current especially around the axis causes the q-profile to change, which might result in a suppression of sawtooth crashes in some regimes. When the turbulence is weaker, i.e., $\tilde{b} \lesssim O\left(10^{-5}\right)$ in the entire plasma region, its effects become less significant and even negligible. A very-broad temperature profile with a steep barrier near the edge has been used to study advanced scenarios. With on-axis turbulence, the equilibrium profiles are not different in a substantial way from the case with no turbulence. The presence of edge-localized turbulence, on the contrary, leads to a notable increase in power (due to anomalous and cross resistivity), while the increase in current is small. Additionally, turbulence produces a bump in the edge current profile, which the hyper-resistive term is not able to smooth out. A fully bootstrapped scenario has been studied by adopting a temperature profile that models a steady-state, fully bootstrapped discharged in the TCV tokamak. This profile leads to a transport barrier which is located closer to the axis. Adopting the on-axis turbulence model, as plausible with this kind of thermodynamic profiles, we find an effective inward diffusion of the current due mainly to the hyper-resistive term. The power balance [Eq. (16)] shows that the power generated internally by the neoclassical and turbulent bootstrap terms can compensate for the dissipation by the remaining terms, thus eliminating the need for an external source.

The theoretical understanding of the influence of turbulence on Ohm's law in tokamaks is a very difficult task, requiring complex kinetic calculation. While we realize that a reasonably accurate and widely accepted theory is still lacking, we also note that there are features that are common to most theories that consider extension of Ohm's law due to turbulent effects. ${ }^{10,45-47}$ We mention the work of Ref. 48, where it is estimated that turbulence can generate a current up to $10 \%$ of the neoclassical bootstrap, a potentiality built in our model too, as the numerical studies of the present work show. In the cited work, the effective electric field (due to turbulence) was proportional to $k_{\|} R_{0}$, and thus, a symmetry breaking mechanism (e.g., a shear flow or an inhomogeneous turbulent intensity) was required in order to have a nonzero contribution. In our current work, we have not invoked a symmetry breaking mechanism, and so, the integration in $k_{\|}$over the normal modes of magnetic turbulence with $k_{\|} \ll k_{\perp}$ cancels out and gives no contribution to the final expression of the turbulent electric field. The overall effects in $k_{\perp}$, which are non-zero, stem from the self-consistency of our theory (as shown in Ref. 18). It is a well-documented fact that retaining 
the friction term in the collision operator could lead, in some cases, to results quite different from the corresponding results obtained with the quasi-linear theory. We cite two examples: Ref. 49, in which the energy exchange between species is evaluated using both the quasi-linear theory and self-consistent theory, and Ref. 50, which shows that self-consistency leads to particle pinches.

One of the setbacks of this work is the use of circular flux surfaces and classical instead of neoclassical resistivity; both approximations are strictly valid for cylindrical plasmas. Even with this simplified model, we were able to recover earlier results and give some new insights into the effects of each turbulent coefficient on the current and magnetic profiles in various scenarios. The turbulent perturbation profile used here is fairly arbitrary, and it could be improved by reverse engineering, i.e., matching the current density experimental data with the theoretical predictions. The first step to expand this work will be to use turbulence profiles generated from a more advanced numerical code. We already mentioned that the current density itself affects the temperature and density profiles and with that the general plasma stability. This is especially important when a large fraction of the current is due to bootstrap, in which case the mutual interaction between the current and thermodynamic profiles must be taken into account selfconsistently. This has not been done here and should be addressed by coupling Ohm's law to a transport code evolving the equilibrium profiles. Finally, in all computations, we have set $j_{\|}=0$ at the plasma outer boundary. Thanks to turbulent diffusion, however, a nonzero current at the plasma edge could potentially sustain a significant fraction of the plasma current. ${ }^{51}$

\section{ACKNOWLEDGMENTS}

Useful discussions with C. Angioni, E. Poli, S. Günter, and P. Lauber are kindly acknowledged. This work was part of Max Planck-Princeton (MPPC) Center for Plasma Physics study 2015-2017 and was partially supported by ITER-CN under Project No. 2015GB110003.

${ }^{1}$ A. A. Galeev and R. Z. Sagdeev, Sov. Phys. JEPT 26, 233-240 (1968).

${ }^{2}$ R. J. Bickerton, J. W. Connor, and J. B. Taylor, Nat. Phys. Sci. 229, 110-112 (1971).

${ }^{3}$ R. H. Weening and A. H. Boozer, "Completely bootstrapped tokamak," Phys. Fluids B 4, 159-170 (1992).

${ }^{4}$ K. Itoh, S.-I. Itoh, and A. Fukuyama, Comments Plasma Phys. Controlled Fusion 15, 151-157 (1992).

${ }^{5}$ A. D. Turnbull, T. S. Taylor, M. S. Chu, R. L. Miller, and Y. R. Lin-Liu, Nucl. Fusion 38, 1467-1486 (1998).

${ }^{6}$ Y. Sakamoto, T. Fujita, S. Ide, M. Takechi, T. Suzuki, A. Isayama, H. Takenaga, N. Oyama, Y. Kamada, and the JT-60 Team, Nucl. Fusion 45, 574-580 (2005).

${ }^{7}$ S. Coda, O. Sauter, M. A. Henderson, and T. P. Goodman, in 22nd IAEA Fusion Energy Conference, Geneva, 13th-18th October 2008, p. EX/2-3.

${ }^{8}$ J. Schmidt and S. Yoshikawa, Phys. Rev. Lett. 26, 753-756 (1971).

${ }^{9}$ D. Biskamp, Plasma Phys. Controlled Fusion 26, 311-319 (1984).

${ }^{10} \mathrm{R}$. Gatto and I. Chavdarovski, "Current density equation in magnetic turbulence,” Open Plasma Phys. J. 4, 1-15 (2011).

${ }^{11}$ W. J. Nunan and J. M. Dawson, Phys. Rev. Lett. 73, 1628-1631 (1994).

${ }^{12}$ T. K. Fowler, Comments kinetic dynamo (1995), UCRL-ID-121728.

${ }^{13}$ A. N. Kaufman, Phys. Fluids 15, $1063-1069$ (1972).

${ }^{14}$ H. E. Mynick and R. E. Duvall, Phys. Plasmas 1, 750 (1989).

${ }^{15}$ S. M. Mahajan, R. D. Hazeltine, and D. A. Hitchcock, Phys. Fluids 26, 700-704 (1983).
${ }^{16}$ R. Gatto and I. Chavdarovski, "Self-consistent electron transport in tokamaks," Phys. Plasmas 14, 092502 (2007).

${ }^{17}$ R. Gatto and I. Chavdarovski, "Magnetic turbulent transport from selfconsistent action-angle theory," in 33rd EPS Conference on Plasma Physics Rome, 19-23 June 2006, Vol. 30I, p. 4.150 (2006).

${ }^{18} \mathrm{I}$. Chavdarovski and R. Gatto, "Turbulent sources in axisymmetric plasmas," Plasma Phys. Controlled Fusion 51, 075002 (2009).

${ }^{19}$ A. B. Rechester and M. N. Rosenbluth, Phys. Rev. Lett. 40, 38-41 (1978).

${ }^{20}$ K. Ida, Y. Miura, T. Matsuda, K. Itoh, S. Hidekuma, S.-I. Itoh, and JFT2M Group, Phys. Rev. Lett. 74, 1990-1993 (1995).

${ }^{21}$ A. H. Boozer, "Ohm's law for mean magnetic fields," J. Plasma Phys. 35, 133-139 (1986).

${ }^{22}$ A. Bhattacharjee and E. Hameiri, Phys. Rev. Lett. 57, 206-209 (1986).

${ }^{23}$ H. K. Moffatt, Magnetic Field Generation in Electrically Conducting Fluids (Cambridge University Press, Cambridge, 1978).

${ }^{24}$ Y. S. Hwang, C. B. Forest, and M. Ono, Phys. Rev. Lett. 77, 3811-3814 (1996).

${ }^{25}$ J. B. Taylor, Phys. Rev. Lett. 33, 1139-1141 (1974).

${ }^{26}$ J. M. Rawls, M. S. Chu, and F. L. Hinton, Phys. Fluids 18, 1160-1166 (1975).

${ }^{27}$ Y. Hamada, T. Watari, A. Nishizawa, O. Yamagishi, K. Narihara, K. Ida, Y. Kawasumi, T. Ido, M. Kojima, K. Toi, and the JIPPT- IIU Group, Nucl. Fusion 55, 043008 (2015).

${ }^{28}$ L. Colas, X. L. Zou, M. Paume, J. M. Chareau, L. Guiziou, G. T. Hoang, Y. Michelot, and D. Grésillona, Nucl. Fusion 38, 903 (1998).

${ }^{29}$ M. C. Firpo, Phys. Plasmas 22, 122511 (2015).

${ }^{30}$ F. L. Hinton, J. C. Wiley, D. F. Düchs, H. P. Furth, and P. H. Rutherford, Phys. Rev. Lett. 29, 698-701 (1972).

${ }^{31}$ R. D. Hazeltine and F. L. Hinton, Phys. Fluids 16, 1883-1889 (1973).

${ }^{32}$ W. W. Pfeiffer, R. H. Davidson, R. W. Miller, and R. E. Waltz, General Atomic Report No. GA-A16178, 1980.

${ }^{33}$ Y. Yuan and A. Bhattacharjee, Phys. Fluids B 5, 3661-3667 (1993).

${ }^{34}$ T. A. Casper, R. J. Jayakumar, S. L. Allen, C. T. Holcomb, L. L. LoDestro, M. A. Makowski, L. D. Pearlstein, H. L. Berk, C. M. Greenfield, T. C. Luce, C. C. Petty, P. A. Politzer, and M. R. Wade, Nucl. Fusion 47, 825-832 (2007).

${ }^{35}$ M. S. Chu, D. P. Brennan, V. S. Chan, M. Choi, R. J. Jayakumar, L. L. Lao, R. Nazikian, P. A. Politzer, H. E. St.John, A. D. Turnbull, M. A. Van Zeeland, and R. White, Nucl. Fusion 47, 434-442 (2007).

${ }^{36}$ J. Garcia and G. Giruzzi, Phys. Rev. Lett. 104, 205003 (2010).

${ }^{37}$ C. C. Petty, M. E. Austin, C. T. Holcomb, R. J. Jayakumar, R. J. La Haye, T. C. Luce, M. A. Makowski, P. A. Politzer, and M. R. Wade, Phys. Rev. Lett. 102, 045005 (2009).

${ }^{38}$ R. Wolf, O. Gruber, M. Maraschek, R. Dux, C. Fuchs, S. Günter, A. Herrmann, A. Kallenbach, K. Lackner, P. J. McCarthyd, H. Meister, G. Pereverzev, J. Schweinzer, U. Seidel, and the ASDEX Upgrade Team, Plasma Phys. Controlled Fusion 41, B93 (1999).

${ }^{39}$ S. Günter, G. Giruzzi, A. Gude, R. J. La Haye, K. Lackner, M. Maraschek, S. Schade, S. Sesnic, R. Wolf, Q. Yu, H. Zohm, and the ASDEX Upgrade Team, Plasma Phys. Controlled Fusion 41, B231 (1999).

${ }^{40}$ S. C. Jardin, N. Ferraro, and I. Krebs, Phys. Rev. Lett. 115, 215001 (2015).

${ }^{41}$ A. G. Peeters, "The bootstrap current and its consequences," Plasma Phys. Controlled Fusion 42, B231-B242 (2000).

${ }^{42}$ J. A. Wesson, Nucl. Fusion 18, 87 (1978).

${ }^{43}$ C. Kessel, J. Manickam, G. Rewoldt, and W. M. Tang, Phys. Rev. Lett. 72, 1212 (1994).

${ }^{44}$ S. Coda, O. Sauter, M. A. Henderson, T. P. Goodman, and the TCV Team, in Proceedings of the 34th EPS Conference on Controlled Fusion and Plasma Physics, Warsaw (2007), Europhysics Conference Abstract 31F (2007) (D-1.008).

${ }^{45}$ S.-I. Itoh and K. Itoh, Phys. Lett. A 127, 267 (1988).

${ }^{46}$ F. L. Hinton, R. E. Waltz, and J. Candy, Phys. Plasmas 11, 2433 (2004).

${ }^{47}$ C. J. McDevitt, X.-Z. Tang, and Z. Guo, Phys. Rev. Lett. 111, 205002 (2013).

${ }^{48}$ X. Garbet, D. Esteve, Y. Sarazin, G. Dif-Pradalier, P. Ghendrih, V. Grandgirard, G. Latu, and A. Smolyakov, J. Phys.: Conf. Ser. 561, 012007 (2014).

${ }^{49}$ H. E. Mynick, Phys. Fluids B 4, 1229-1237 (1992).

${ }^{50}$ R. Gatto, Nucl. Fusion 47, L1-L4 (2007).

${ }^{51}$ M. Ono, G. J. Greene, D. Darrow, C. Forest, H. Park, and T. H. Stix, Phys. Rev. Lett. 59, 2165-2168 (1987). 